\title{
Correlates of hepatitis B awareness and disease-specific knowledge among pregnant women in Northern and Central Uganda: a cross-sectional study
}

\author{
Joan Nankya-Mutyoba ${ }^{1 *}$ D, Jim Aizire ${ }^{2}$, Fredrick Makumbi ${ }^{1}$, Lynn Atuyambe ${ }^{3}$, Ponsiano Ocama ${ }^{4}$ and Gregory D. Kirk ${ }^{2,5}$
}

\begin{abstract}
Introduction: Countries in sub-Saharan Africa with a high hepatitis B burden also have limited resources to identify underlying drivers of disease among key at-risk populations. To improve prioritization and strengthen prevention of mother to child transmission of HBV, it is imperative to understand disease awareness, knowledge and related factors among pregnant women.
\end{abstract}

Objectives: This study assessed HBV disease awareness, knowledge and related factors among pregnant women in public health facilities in two regions with diverse HBV disease epidemiology.

Methods: From October 2016 through December 2017, a random sample of 455 pregnant women attending antenatal clinics were surveyed to assess HBV awareness, knowledge and associated factors. Participants responded to an 18-item questionnaire with themes on HBV awareness, knowledge of disease signs and symptoms, transmission, prevention and misconceptions about the disease. Results were analysed in STATA (version 14.0).

Results: Of 455 participants enrolled, about two thirds reported having heard about HBV disease. By region, nearly half (47\%) of participants from the central region, compared to only $16 \%$ from the north, reported that they had never heard of HBV. Region of residence had a moderating effect on the education- HBV awareness relationship. Only 162/455 (36\%) of participants had adequate HBV knowledge. More than half 256/455 (56\%) and 242/455 (53\%) were not knowledgeable about horizontal and mother to child HBV transmission, respectively. About two thirds 298/455 (66\%) and 281/455 (62\%) believed HBV was spread via sharing of utensils and mosquito bites respectively. In multiple regression analysis, residing in the north, $(P R=1.91(1.53-2.38), p<0.001)$ compared to central region and having a secondary education ( $P R=1.87(1.37-2.55), p<0.001)$ compared to primary were statistically significantly related to being knowledgeable about HBV.

Conclusion: We demonstrated marked regional differences in HBV disease awareness and knowledge in this high HBV prevalence setting. However, most pregnant women displayed unacceptably low HBV knowledge and a significant proportion still hold misconceptions about HBV. Interventions to improve HBV prevention through antenatal education will need to be tailored to existing differences in comprehensive HBV knowledge.

Keywords: Hepatitis B, Awareness, Knowledge, Pregnant Women

\footnotetext{
* Correspondence: eron.jm@hotmail.com

'Department of Epidemiology \& Biostatistics, School of Public Health,

Makerere University College of Health Sciences, P.O. Box 7072, Kampala,

Uganda

Full list of author information is available at the end of the article
}

(c) The Author(s). 2018 Open Access This article is distributed under the terms of the Creative Commons Attribution 4.0 International License (http://creativecommons.org/licenses/by/4.0/), which permits unrestricted use, distribution, and reproduction in any medium, provided you give appropriate credit to the original author(s) and the source, provide a link to the Creative Commons license, and indicate if changes were made. The Creative Commons Public Domain Dedication waiver (http://creativecommons.org/publicdomain/zero/1.0/) applies to the data made available in this article, unless otherwise stated. 


\section{Introduction}

Chronic Hepatitis B virus (HBV) infection is among the commonest infections world-wide, with an estimated affected population of over 240 million individuals [1]. Chronic infection with HBV places these individuals at increased risk of death from cirrhosis and liver cancer [2-4]. A significant portion of Africa's HBV burden is carried in sub-Saharan African (SSA) nations [5]. Uganda has recorded a much higher HBV burden than other countries within the East African sub-region, with national estimates averaging $10 \%$ and notable regional variations in disease burden [6, 7]. A recent study using modelling and expert opinion reported Uganda to have an estimated HBV prevalence of $5.5 \%$ and $1.5 \%$ among adult and 5-year old populations respectively [8]. Surveys conducted in two important sub-populations i.e. health care workers $[9,10]$ and pregnant women [11] have found HBV infection prevalence above $10 \%$, although an earlier study [12] among HIV-infected pregnant women from a private, not-for-profit hospital had reported hepatitis B surface antigen positivity of $4.9 \%$. It remains uncertain however, whether disease awareness in this population sub-group is adequate and whether variations in knowledge gaps mirror those observed in disease burden. The response to viral hepatitis at national program level in Uganda has been modest [13-16]. Like most SSA countries, routine HBV vaccination of infants before one year is high, at $93 \%$, but only $3 \%$ of infected pregnant women eligible for antiviral therapy have been initiated on treatment. Screening of women for HBV during pregnancy, plus follow-up care including full vaccination, treatment with hepatitis B immunoglobulin and birth dose vaccinations yet to be implemented in public health care facilities in Uganda [8]. In addition, there is inadequate research evidence on HBV disease epidemiology and population-level knowledge and awareness. While HBV may be known as infectious and cancer-causing within the medical and scientific community, it remains unclear how much knowledge exists in the general population, to which pregnant women mostly belong. The first global health sector strategy on viral hepatitis by the world health organization (WHO) to eliminate HBV [17] as a major global health threat by 2030, calls for information that is usable for action, including increase in access to HBV vaccinations and general disease awareness in populations. This current gap in HBV research contributes a barrier to health system strengthening for HBV elimination.

Pregnant women remain an important sub-population for prevention of mother to child transmission of $\mathrm{HBV}$ $[3,18]$. In countries where antenatal HBV prevalence is high, they represent a population sub-group to be targeted for $\mathrm{HBV}$ micro-elimination. If pregnant women have accurate information on $\mathrm{HBV}$ transmission and prevention through vaccination, they are more likely to engage with the health care system, to actively seek and get testing services and ensure their unborn babies are protected, as studies in similar infectious diseases have shown $[19,20]$. Yet there is limited documentation of the extent of awareness and specific knowledge about $\mathrm{HBV}$, its transmission or prevention, among pregnant women. Within SSA, the few studies to evaluate disease awareness and knowledge among pregnant women have been mostly conducted in West [13, 14] and Central Africa $[15,16]$, which have different cultures and disease burden. We conducted a survey among pregnant women attending routine antenatal clinics in public health facilities in 2 out of 4 regions of Uganda, namely Central and Northern regions, with the objective of measuring hepatitis $\mathrm{B}$ prevalence and risk factors and assessing HBV and liver cancer knowledge, perceptions and prevention intentions in this obstetric population. The two regions have different epidemiologic profiles of $\mathrm{HBV}$ with prevalence ranging from $6 \%$ in the Central region and close to $20 \%$ in the Northern region [10]. This analysis focuses on HBV awareness and knowledge.

\section{Methods}

\section{Study setting}

This was a cross-sectional study conducted in public health care facilities in central and northern regions. In the northern region, the study site was Arua Hospital, a regional referral hospital located in Arua town, about 300 miles north-west from Kampala city. It is a high volume, 323 bed-capacity hospital that serves an estimated population of 782,077 covering districts of West Nile and parts of Northern Uganda. It receives about 153,451 out-patients and 5,149 antenatal clinic attendees per year [21]. In the central region, study sites were Kiswa health center III and Kasangati health center IV, both high volume primary care health facilities [22] located in suburban areas in Kampala and Wakiso districts. Both health centers receive 850-1,000 antenatal attendees per month, on average.

\section{Study population}

For inclusion, eligible participants had to be pregnant, attend their routine antenatal clinic visit, have a medical file with the health facility and be at least 18 years of age. Participants who did not provide written informed consent, plus those who could not understand the interviewing language or who were too ill to undergo study-specific procedures were excluded.

\section{Sample selection}

We used Kish \& Leslie formula [23] for sample size estimation, for an estimated knowledge prevalence of $50 \%$ and a $5 \%$ error rate. We included a non-response fraction of $10 \%$, and obtained a total sample size of 455. Participants were sampled using a two stage-sampling 
approach. In the central region, all health center III and IV facilities were enumerated, and stratified by urban or semi-urban status, the divide based on population served $[24,25]$. Then, one health facility was randomly selected from each group, i.e. Kiswa and Kasangati health centers, both of which serve urban and semi-urban populations in different localities of the central region. In the North-west, Arua hospital was selected, considered to serve both urban and semi-urban populations in this region.

Pregnant women were recruited from antenatal clinics on Mondays, Tuesdays and Thursdays in Kiswa and Kasangati health units, and on Mondays and Thursdays in Arua, as these were days when general antenatal clinics operated. All women were generally informed about the study during antenatal education sessions. Then, women to participate were systematically sampled; every $5^{\text {th }}$ woman waiting in line to be seen was approached and informed about the study in detail, and informed consent was sought and obtained prior to study enrollment. Some women preferred to go through the study procedures before proceeding to complete their clinical evaluation, while others preferred to complete study procedures after completion of their routine antenatal visit process.

\section{Data collection}

Trained nurses or midwives were trained as study personnel. They underwent a 3-day training on studyspecific procedures, HBV facts, interviewing techniques, ethical issues and data documentation. In addition, they each completed a certified $\mathrm{NIH}$ online ethics training course. One of the training days was used to pilot study tools, using clinic nurses and pregnant women from nearby health units that would not be used for data collection. Questionnaires were refined according to feedback received.

Questionnaires were administered to every consenting woman to document data on HBV and liver cancer awareness and knowledge, perceptions and preventive behavioral intentions. Questions on knowledge inquired about whether participants had ever heard of HBV disease, its signs and symptoms, and body part affected. Questions on routes of transmission asked whether participants knew that HBV is transmitted sexually, or from mother to child during child birth, or by sharing of needles, or by contact with infected body fluids, through practices like oral warming or pre-chewing of children's food. Other questions asked about routes of transmission that have no bearing to HBV. Questions on prevention asked whether participants knew that HBV or liver cancer can be prevented with a vaccine. Information on socio-demographic factors (age, religion, marital status, highest education achieved, region where one was born, region where one has lived for past 12 months) was also obtained from participants.

\section{Measures}

\section{Hepatitis $B$ disease awareness}

We assessed HBV disease awareness using two approaches. First, using one question "Have you ever heard of a disease called hepatitis B?" (response as "Yes"/“No"), the proportion of participants who responded "Yes" and "No" were classified as being "aware" and "Unaware" of HBV disease, respectively. In the second approach, to further measure disease awareness, we inquired from those participants who responded "yes" to the question "Have you ever heard about hepatitis B?", 3 more questions; "Are you aware of which body organ gets affected by hepatitis $B$ ? Are you aware of any sign or symptom of hepatitis $B$ ? and are you aware of any route by which hepatitis $B$ may be spread?", to which participants had to mention a body part affected, a sign or symptom and one route or method of disease spread, respectively and responses were documented. Participants with correct responses to at least two of the three questions were then further classified as having "high awareness", else they were classified as having "low awareness".

\section{Knowledge of $\mathrm{HBV}$}

In order to further evaluate for more comprehensive HBV disease knowledge, participants were asked 10 questions which inquired about HBV transmission routes (4 questions), prevention (2 questions) and common misconceptions (4 questions) about the disease and its causation. For each question item, responses were either "Yes/No/Don't know" or "True/False/Don't know". Each correct response was assigned a score of ' 1 ' while each incorrect response was assigned a score of ' 0 '. A composite variable of knowledge scores was generated. Two cut off values of knowledge scores were evaluated for possible cut off for adequate knowledge, 7/10 and 8/ 10. The cut off score of $7 / 10$ was considered to be justifiable, for the extent of difficulty of the questions and the study population. It was comparable [26, 27], and in some instances more stringent [28] than cut off values of studies in similar populations elsewhere.

A cut off value of $7 / 10$ correct responses was therefore defined as "adequate knowledge" and scores below this value as "inadequate knowledge". Two questions were not included in the score for knowledge variable, as these were considered as knowledge to be expected from individuals with a medical training, yet the study population were lay persons.

\section{Socio-demographic variables}

Given the narrow age range, age was classified into three categories of under 20 years, 20-24 years and older than 24 years, according to categorizations of the Uganda demographic and health survey. This categorization also enabled examination of the relationship between 
adolescent compared to older pregnant women, regarding HBV disease awareness and knowledge. Three nominal categories of religion were created (catholic, protestant and Islam/other). Likewise, region of birth was categorized into three groups, "North", "Central", and participant who reported have been born in regions besides these two, were classified as "other". Individuals born outside the two study regions were few, and these were merged to form the third category. Education was grouped into two, primary and secondary, as very few individuals had post-secondary education. Status of marriage was also categorized into monogamous, polygamous and divorced/single/other. The third category was merged into one group due to very few participants who reported being single, divorced and/or separated.

\section{Data analysis}

Raw data was entered into excel and data cleaning done before it was exported to STATA (version 14.0) for analysis. Descriptive and stratified analyses were done and data summarized as means with corresponding standard deviation (sd) for continuous variables and proportions for categorical variables. Chi-square for difference in proportions were done. Bivariate analyses were done to estimate associations between individual factors with (i) HBV awareness (ii) HBV knowledge.

Variables that were significant at a $p$-value of 0.20 at bivariate level, plus those considered important based on plausibility were entered into a multivariable model. We used a modified Poisson regression model with robust variance estimation to obtain prevalence rate ratios $[29,30]$ with $95 \%$ confidence intervals, given that the outcomes had a prevalence greater than $10 \%$. In adjusted models for HBV awareness, we estimated the effect of region of residence, on the association between education and HBV awareness. This was estimated from summing up the coefficients of education level and the interaction term, from the multivariable model. The summed up coefficient was then exponentiated to obtain the prevalence risk ratio for this stratum. Education had a moderating effect on the observed relationship between region of residence and HBV disease awareness, which was computed using a similar method. A p-value of 0.05 was used as a cut off for statistical significance. To further evaluate disease awareness and knowledge, we constructed a binary "awareness \& knowledge" variable, which was defined as being both aware of, and having comprehensive HBV knowledge as initially defined as a knowledge score of at least 7 out of 10, "adequate knowledge", or being neither aware of, nor having comprehensive hepatitis B knowledge. Factors associated with the composite "awareness \& knowledge" variable were evaluated in both bivariable and multivariable models.

\section{Results}

A total of 455 participants who were approached consented to study participation and completed the questionnaire, 155 from the northern region and 300 from the central region antenatal clinics, giving a response proportion of $100 \%$. About 45 participants opted to complete the survey in English, instead of the local language (Lugbara, Northern region and Luganda, Central region).

\section{Pregnant women's socio-demographic characteristics}

The socio-demographic attributes of participants are shown in table 1 . The median age was 24 years $(\mathrm{IQR}=21-28)$ and $14 \%$ of respondents were adolescents in both regions. A higher proportion of participants were from the central region, who contributed 300 (65.9\%) of the total sample, while participants from the north contributed 155 (34.1\%) of the sample.

Two thirds of participants, 294/455 (65.3\%), had achieved at least a secondary-level education. Marital unions were reported as mostly monogamous in the north, $130 / 155$ (84.4\%), but mostly polygamous in the central region, 202/300 (67.3\%) and almost half of participants in the north were catholic, (43.0\%) while half of participants in the central region were protestant, 152/300 (50.7\%).

\section{Awareness of HBV}

Overall, about two thirds, 285/453, (62.9\%) of participants had ever heard about HBV disease. By region, only about half, 157/300 (52.3\%) of participants from the central region had ever heard of HBV, compared to majority, 128/ 153, (83.7\%) participants from the North. Additionally, among those overall who had ever heard about HBV, more than two thirds, $182 / 285$ (63.9\%) had low awareness of HBV. Within the northern region, three quarters of participants, 96/128 (75.0\%), had low HBV awareness, versus slightly over half, 86/157 (54.8\%) of central region participants (Fig. 1). Only a quarter (25\%) of participants from the north had high HBV disease awareness, compared to $45 \%$ of those from the central region.

Figure 1 Flowchart showing numbers and proportions of respondents, by geographic region (North and Central) who reported having heard (Awareness) or never having heard (No Awareness) about HBV, plus those who had low and high HBV awareness, among those who reported that they were aware about HBV. Total N=Total number of participants

About half $(49 \%)$ of adolescent pregnant women reported that they had never heard about HBV, compared to only $35 \%$ of the older women. Among pregnant adolescents who reported having heard of HBV, 20\% (compared to $43 \%$ of older women) had low awareness of HBV. Overall, majority of respondents $(77.4 \%)$ were either completely unaware of HBV disease, or only heard 
Table 1 Socio-demographic characteristics of study participants, overall and according to site of recruitment.

\begin{tabular}{|c|c|c|c|c|c|}
\hline Characteristic & & Total $N=455$ & North $N=155$ (34.1\%) & Central $N=300$ (65.9\%) & $p$-value* \\
\hline Age (years)Median (IQR) & & $18-45$ & $18-42$ & $18-45$ & \\
\hline \multirow[t]{3}{*}{ Age group (years), n (\%) } & $<20$ & $65(14.4)$ & $22(14.2)$ & $43(14.4)$ & 0.477 \\
\hline & $20-24$ & $174(38.4)$ & $54(34.8)$ & $120(40.3)$ & \\
\hline & $>24$ & $214(47.2)$ & $79(51.0)$ & $135(45.3)$ & \\
\hline \multirow[t]{2}{*}{ Education level, n (\%) } & $\leq$ Primary & $156(34.7)$ & $57(36.8)$ & 99 (33.6) & 0.496 \\
\hline & $\geq$ Secondary & $294(65.3)$ & $98(63.2)$ & $196(66.4)$ & \\
\hline \multirow[t]{3}{*}{ Region of birth, n (\%) } & North & $136(30.0)$ & $129(83.8)$ & $7(2.34)$ & $<0.001$ \\
\hline & Central & $168(37.1)$ & $2(1.3)$ & $166(55.5)$ & \\
\hline & Other & $149(32.9)$ & $23(14.9)$ & $126(42.1)$ & \\
\hline \multirow[t]{3}{*}{ Marital status, n (\%) } & Single/divorced & $24(5.2)$ & $5(3.25)$ & $19(6.3)$ & $<0.001$ \\
\hline & Monogamy & $209(46.0)$ & $130(84.4)$ & $79(26.3)$ & \\
\hline & Polygamy & $122(48.7)$ & 19 (12.3) & $202(67.3)$ & \\
\hline \multirow[t]{3}{*}{ Religion, n (\%) } & Catholic & $157(34.5)$ & $66(42.6)$ & $91(30.3)$ & 0.002 \\
\hline & Protestant & $204(44.8)$ & $52(33.5)$ & $152(50.7)$ & \\
\hline & Islam/other & $94(20.7)$ & $37(23.9)$ & $57(19.0)$ & \\
\hline
\end{tabular}

${ }^{*} p$-value $=$ chi-square

of the disease, but were unaware of its signs, symptoms or how it is spread.

\section{Knowledge of HBV}

Using a defined cut off score for adequate HBV knowledge, only about $36 \%(162 / 455)$ of participants were knowledgeable about HBV, of which $60 \%$ (98/162) were from the central region. Figure 2 provides details about components of HBV knowledge (transmission, prevention, complications and myths related to causes). More than two thirds of all participants (69.2\%) knew that
HBV infection is preventable by vaccination. Approximately half the surveyed population knew that HBV infection can result in liver cancer and that this can be prevented by HBV vaccination (51\%), were knowledgeable about sexual (54\%) and needle-sharing (56\%) routes of HBV transmission. Fewer participants overall, knew that HBV can be spread to the unborn baby by infected mother during child birth (46.8\%), or to the newborn via horizontal transmission (43.7\%), when newborn may come into contact with blood or body fluids of an infected adult within the household

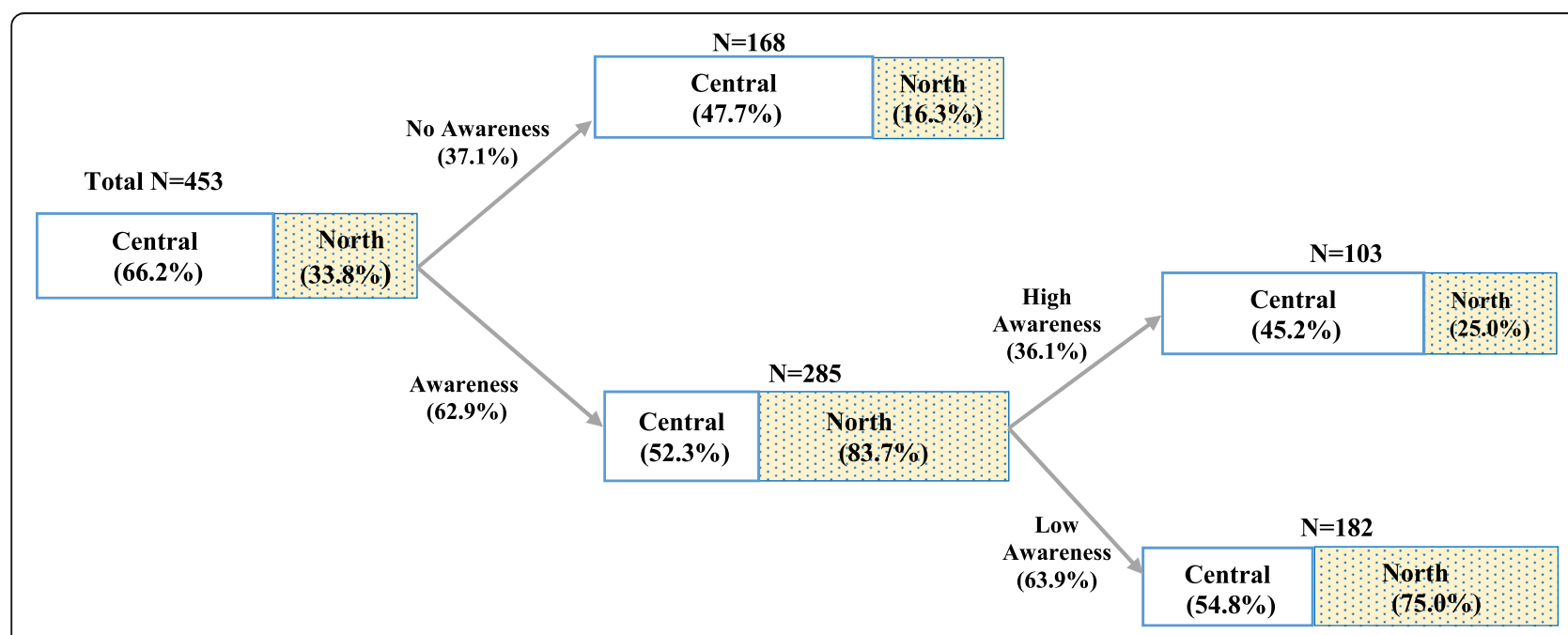

Fig. 1 Proportion of women with no awareness, low awareness and high awareness of hepatitis B 


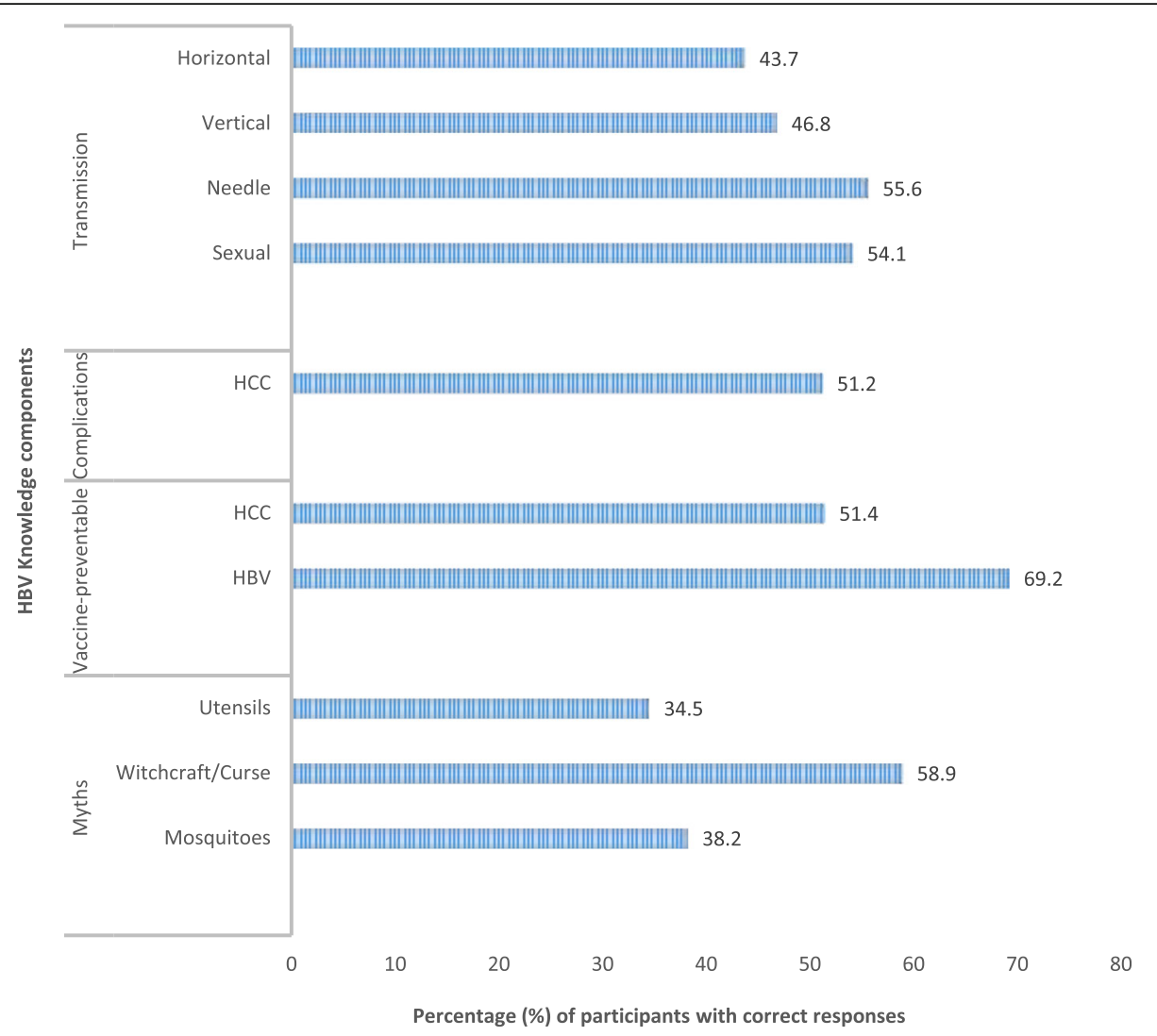

Fig. 2 Distribution of proportion of women with correct responses about different hepatitis B knowledge components

environment. Knowledge about sexual route of transmission was more prevalent in the Northern region $(70.3 \%)$ compared to the central region, (45.7\%) while knowledge about horizontal transmission was more prevalent in the central region,(50\%). In both regions, only about half, (Central region $44.7 \%$, Northern region $51.3 \%$ ) of participants were knowledgeable about mother-to-child transmission of HBV. Regarding misconceptions, a minority of participants knew that HBV is not spread by mosquito bites (38.2\%), or by sharing cooking utensils $(34.5 \%)$ and a sizeable proportion (41.0\%) believed the disease can be caused by a curse or witchcraft (Fig. 2).

Figure 2 Proportion of respondents with correct responses to questions on routes of HBV transmission, complications, vaccine prevention and myths regarding causes. HCC=hepatocellular carcinoma or liver cancer. $\mathrm{HBV}=$ hepatitis $\mathrm{B}$ virus.

\section{Correlates of HBV disease awareness}

In both unadjusted and adjusted models (Table 2.0; model 1 and table 3.0; model 1, respectively) region of residence, age and education were related to HBV awareness. Region of residence had a moderating effect on the observed relationship between education and HBV awareness. Among participants residing in the central region, having a secondary education was associated with $76 \%$ greater prevalence of $\mathrm{HBV}$ awareness compared to having a primary or lower level of education ( $\mathrm{PRR}=1.76[95 \% \mathrm{CI}=1.32-2.35] p<0.001)$, whereas among participants residing in the north, having a secondary education, compared to primary or lower level was associated with only $25 \%$ greater prevalence of $\mathrm{HBV}$ awareness, adjusting for age (PRR=1.25). Similarly, education had a moderating effect on the observed relationship between region of residence and HBV disease awareness. Among participants with primary or lower level of education, residents of northern region had a prevalence of HBV awareness that was twice that of central region residents $(\mathrm{PRR}=2.04[95 \% \mathrm{CI}=1.49-2.80]$ $p<0.001$ ), while among participants with a secondary or higher level of education, residents of the north, compared to central region had only $45 \%(P R R=1.45)$ greater HBV disease awareness prevalence, adjusting for age. Pregnant women older than 24 years had a prevalence of HBV awareness that was $33 \%$ greater than that of women aged under 20 years $(\mathrm{PRR}=1.33[1.03-1.70] p=0.026)$ and this association was statistically significant. In bivariate analyses, being born in the north compared to elsewhere $(\mathrm{PRR}=1.48(1.26-1.73), p<0.001)$ was associated with a prevalence of HBV awareness that was $48 \%$ higher. 
Table 2 Bivariable regression analysis of factors associated with hepatitis B virus awareness and knowledge^

\begin{tabular}{|c|c|c|c|c|c|c|c|c|c|}
\hline \multirow[t]{2}{*}{ Factor } & \multicolumn{3}{|l|}{ Model $1^{a}$} & \multicolumn{3}{|l|}{ Model $2^{b}$} & \multicolumn{3}{|l|}{ Model $3^{c}$} \\
\hline & Unadjusted PRR & $95 \% \mathrm{Cl}$ & $p$-value & Unadjusted PRR & $(95 \% \mathrm{Cl})$ & $p$-value & Unadjusted PRR & $(95 \% \mathrm{Cl})$ & $p$-value \\
\hline \multicolumn{10}{|l|}{ Age group (years) } \\
\hline$<20$ & 1 & & & 1 & & & 1 & & \\
\hline $20-24$ & 1.19 & $(0.91-1.56)$ & 0.214 & 1.27 & $(0.81-2.01)$ & 0.302 & 1.13 & $(0.76-1.66)$ & 0.553 \\
\hline$>24$ & 1.35 & $(1.04-1.75)$ & 0.023 & 1.41 & $(0.90-2.21)$ & 0.129 & 1.05 & $(0.71-1.55)$ & 0.804 \\
\hline \multicolumn{10}{|l|}{ Education level } \\
\hline$\leq$ Primary & 1 & & & 1 & & & 1 & & \\
\hline$\geq$ Secondary & 1.47 & $(1.23-1.75)$ & $<0.001$ & 1.40 & $(1.05-1.86)$ & 0.021 & 1.40 & $(1.05-1.86)$ & 0.021 \\
\hline \multicolumn{10}{|l|}{ Region of birth } \\
\hline Other & 1 & & & 1 & & & 1 & & \\
\hline North & 1.48 & $(1.26-1.73)$ & $<0.001$ & 1.91 & $(1.43-2.56)$ & $<0.001$ & 1.42 & $(1.04-1.94)$ & 0.026 \\
\hline Central & 0.89 & $(0.72-1.09)$ & 0.250 & 0.97 & $(0.68-1.38)$ & 0.875 & 1.14 & $(0.83-1.56)$ & 0.427 \\
\hline \multicolumn{10}{|l|}{ Religion } \\
\hline Islam/other & 1 & & & 1 & & & 1 & & \\
\hline Protestant & 0.90 & $(0.75-1.09)$ & 0.283 & 0.81 & $(0.58-1.13)$ & 0.208 & 0.88 & $(0.63-1.22)$ & 0.450 \\
\hline Catholic & 1.03 & $(0.85-1.24)$ & 0.791 & 1.04 & $(0.76-1.43)$ & 0.808 & 1.05 & $(0.76-1.46)$ & 0.759 \\
\hline \multicolumn{10}{|l|}{ Marital status } \\
\hline Single/Divorced & 1 & & & 1 & & & 1 & & \\
\hline Monogamy & 1.37 & $(0.95-1.98)$ & 0.091 & 1.34 & $(0.71-2.54)$ & 0.360 & 1.03 & $(0.63-1.70)$ & 0.897 \\
\hline Polygamy & 0.88 & $(0.60-1.29)$ & 0.500 & 0.67 & $(0.34-1.30)$ & 0.232 & 0.71 & $(0.43-1.19)$ & 0.198 \\
\hline \multicolumn{10}{|l|}{ Residence region } \\
\hline Central & 1 & & & 1 & & & 1 & & \\
\hline North & 1.60 & $(1.41-1.82)$ & $<0.001$ & 1.97 & $(1.57-2.47)$ & $<0.001$ & 1.32 & $(1.03-1.68)$ & 0.029 \\
\hline
\end{tabular}

Model $1^{\mathrm{a}}$ Outcome is "HBV awareness". Model $2^{b}$ Outcome is "Adequate HBV Knowledge". Model $3^{c}$ Outcome is composite score of "Awareness or Knowledge"

$\wedge$ Knowledge $=\mathrm{A}$ score of at least $7 / 10$. $P R R=$ Prevalence rate ratio

Table 3 Multivariable regression analysis of factors associated with hepatitis B awareness and knowledge^

\begin{tabular}{|c|c|c|c|c|c|c|c|c|c|}
\hline \multirow[t]{2}{*}{ Factor } & \multicolumn{3}{|l|}{ Model 1} & \multicolumn{3}{|l|}{ Model 2} & \multicolumn{3}{|l|}{ Model 3} \\
\hline & Adjusted PRR & $(95 \% \mathrm{Cl})$ & $p$-value & Adjusted PRR & $(95 \% \mathrm{Cl})$ & $p$-value & Adjusted PRR & $(95 \% \mathrm{Cl})$ & $p$-value \\
\hline \multicolumn{10}{|l|}{ Age group (years) } \\
\hline$<20$ & 1 & & & 1 & & & 1 & & \\
\hline $20-24$ & 1.14 & $(0.88-1.48)$ & 0.308 & 1.09 & $(0.74-1.61)$ & 0.652 & 1.22 & $(0.81-1.84)$ & 0.349 \\
\hline$>24$ & 1.33 & $(1.03-1.70)$ & 0.026 & 1.04 & $(0.71-1.52)$ & 0.856 & 1.32 & $(0.89-1.98)$ & 0.172 \\
\hline \multicolumn{10}{|l|}{ Education level } \\
\hline$\leq$ primary & 1 & & & 1 & & & 1 & & \\
\hline$\geq$ Secondary & 1.76 & $(1.32-2.35)$ & $<0.001$ & 1.39 & $(1.05-1.85)$ & 0.023 & 1.87 & $(1.37-2.55)$ & $<0.001$ \\
\hline \multicolumn{10}{|l|}{ Region of residence } \\
\hline Central & 1 & & & 1 & & & 1 & & \\
\hline North & 2.04 & $(1.49-2.80)$ & $<0.001$ & 1.33 & $(1.04-1.70)$ & 0.023 & 1.91 & $(1.53-2.38)$ & $<0.001$ \\
\hline Education*Residence & 0.71 & $(0.51-0.99)$ & 0.048 & -- & -- & & --- & --- & \\
\hline
\end{tabular}


However, this variable highly correlated with region of residence, and was dropped from the multivariable models. Marital status and religion were unrelated to HBV disease awareness.

\section{Correlates of HBV knowledge}

Prevalence of adequate HBV knowledge, defined as a knowledge score of at least $7 / 10$, was $35.5 \%(162 / 455)$. In both unadjusted and adjusted models, region of residence and education were related to having comprehensive knowledge of HBV. In multivariable models, participants residing in the northern region had a statistically significant 33\% increase in prevalence of adequate knowledge ( $\mathrm{PRR}=1.33(1.04-1.70), p=0.023)$ compared to those in the central region. Pregnant women with a secondary or higher level of education had a statistically significant $39 \%$ increase in prevalence of adequate comprehensive knowledge of HBV (PRR=1.39 (1.05 -1.85), $p=0.023)$, compared to women with a primary or lower level of education. Age, religion, and marital status were not related to having comprehensive HBV knowledge. Region of birth was dropped from the models due to correlation with region of residence, as most participants tended to reside in the same region they were born. In models without region of residence, region of birth was statistically significantly related to comprehensive HBV knowledge. Factors associated with the composite "awareness \& knowledge" variable are shown in Table 2.0, model 3 and table 3.0, model 3. Region of residence $(\mathrm{PRR}=1.91(1.53-2.38), \quad p<0.001)$ and education $(\mathrm{PRR}=1.87(1.37-2.55), p<0.001)$, emerged as significantly related to being aware and knowledgeable about HBV. There was no significant interaction between education, region of residence and being aware of or having HBV knowledge.

\section{Discussion}

We found a low level of HBV disease awareness with pregnant adolescents tending to have lower HBV awareness, and even lower levels of adequate HBV and liver cancer knowledge in this obstetric population of a high HBV prevalence country, which also records one of the highest $\mathrm{HBV}$-associated liver cancer rates in the world [31]. Higher education, and residing in the northern region, compared to the central region, were associated with being knowledgeable about HBV. Awareness measured using a single question was moderate, but further assessment of awareness revealed that a majority of respondents had low awareness of HBV signs or symptoms, body part mostly affected and at least one method by which the disease spreads. The WHO global health sector strategy on viral hepatitis [17] recognizes the need for critical, indigenous information to direct prevention interventions in different localities and to utilize opportunities like prevention of mother to child HBV transmission through antenatal testing of pregnant women. A key barrier to achieving these goals is low population awareness of $\mathrm{HBV}$, with under $5 \%$ of chronic hepatitis-affected persons aware of their infection status [32]. This, to our knowledge, is one of very few studies to systematically evaluate population $\mathrm{HBV}$ and liver cancer-related awareness and knowledge in sub-Saharan Africa, and can contribute to locally-derived evidence to support HBV and liver cancer prevention efforts. Younger age in this study was associated with lower disease awareness and unrelated to comprehensive HBV knowledge, a finding that differs from a recent study in India [33], where younger people tended to have higher HBV knowledge. While this might be reflective of the younger generation access to information via technology in India, the observed lack of association in our population may be due to lower access to technology-transmitted knowledge than in India. Although about two thirds of the women knew about HBV prevention using a vaccine, a finding similar to that among Chinese pregnant women [34], a smaller proportion in this study was knowledgeable about mother to child HBV transmission and liver cancer as a possible consequence of chronic infection, compared to Chinese pregnant women. We also found a high proportion of women with inaccurate beliefs about HBV causation and transmission, compared to a Cameroonian study [28] in which prevalence of misconceptions was only $2.3 \%$, but comparable to $66.5 \%$ of 504 pregnant women in a Ghanaian study [35]. Another important area where we found deficient knowledge was horizontal HBV transmission, where an infected adult may transmit to an infant through practices such as oral pre-warming or pre-chewing of infants' food by adults, which may involve transmission through saliva or infected body fluids. Several studies [36-39] show evidence of horizontal HBV transmission through saliva, and this practice is common in developing country cultures [40-42], where it has been linked to HIV transmission, yet there is sparse data on knowledge of this route of HBV transmission among pregnant women. In cultures where these practices continue, specific attention will need to be given to effectively communicate horizontal route of transmission, as an effort to strengthen prevention. Both earlier [13] and more recent reviews [14, 15] of HBV transmission and prevention in different countries in SSA and East Africa region [16] did highlight the need to curb vertical and horizontal HBV transmission through provision of both routine HBV infant vaccination and neonatal HBV vaccination at birth, with detailed critique of existing challenges and opportunities therein. The scope of this discourse however, did not capture the wider community-level barriers, including HBV disease awareness or knowledge within the end-user communities, which this study has attempted to do. 
Education was observed to be related to both HBV disease awareness and knowledge in this study. Most of our respondents had either a primary $(31 \%)$ or a secondary (53\%) education, with only $11 \%$ having a post-secondary education. The effect of education on HBV awareness was moderated by region of participants' residence, with the result that for dwellers of the central region, having a secondary education conferred a $76 \%$ increase in prevalence ratios for HBV awareness, compared to only $25 \%$ increase, for northern region dwellers. This observation could be explained by the fact that in Uganda, HBV is more common in the north than the central region, and as such, residents of the north, regardless of their education level, are more likely to have heard about the disease through social networks of family and community than residents of the central region. Conversely, for residents of the central region, HBV disease awareness seems to be propagated more through years of formal literacy, than social networks, since the disease is less prevalent and as such, much less commonly reported in this region. The effect of years of formal education on comprehensive HBV knowledge, was not modified by region of residence. This finding is consistent with findings among pregnant populations within SSA [28, 43].

There were geographic differences in knowledge, with residents of northern region more likely to have heard of $\mathrm{HBV}$, and to have adequate knowledge, and the association was consistent across the 3 models. HBV infection prevalence varies in Uganda, with the northern region having generally higher disease burden [6], which may explain higher odds of having heard of disease, despite significant deficiencies in disease-specific knowledge.

These findings have implications regarding the hepatitis control policy in Uganda and similar SSA settings; $\mathrm{HBV}$ prevention is done through HBV vaccination of infants as part of routine immunization. Interventions like antenatal HBV education, testing or vaccination for pregnant women, neonatal HBV vaccination at birth are lacking. This translates into gaps in HBV services for pregnant women and in locally-derived information such as HBV-status awareness among pregnant women. Comprehensive hepatitis education and accurate information will need to target the observed knowledge deficiencies and adequately attend to them.

This study had important strengths. Our method used multiple approaches to examine awareness and knowledge and we performed multiple sensitivity analyses to examine factors associated with disease awareness and knowledge, using an adequate sample size, to provide reliable estimates of awareness and knowledge, which can precisely inform HBV and liver cancer education interventions. Notable limitations of this work include the self-reported measures, as well as subjective measurement of disease awareness and knowledge which may carry inherent information bias. We attempted to minimize the effect of this subjectivity by constructing another variable that merged awareness of $\mathrm{HBV}$ disease and specific HBV knowledge, comparing those who scored "Low" on both with those who scored "high" on both, across a set of variables of interest.

\section{Conclusion}

We have identified important knowledge deficiencies and misconceptions in relation to $\mathrm{HBV}$ among pregnant women. Even with geographical differences in disease awareness, both regions have critical information gaps that require adequate HBV prevention education policies, placing specific attention to women with fewer than 7 years of formal education, and adolescent pregnant women. Increasing HBV awareness and knowledge in obstetric populations is a critical component of HBV micro-elimination strategies that will lead to elimination of mother to child transmission of HBV by 2030. Accurately informed women may be more likely to seek and access testing and vaccination during pregnancy, as well as targeted birth dose vaccination for exposed newborns, if they have timely access to appropriate education messages. Therefore, designing education messages tailored to observed findings, in order to raise disease awareness and knowledge among pregnant women may be a key initial step towards micro-elimination in this territory.

\section{Abbreviations \\ HBV: Hepatitis B Virus; PR: Prevalence ratio.; SD: Standard deviation; SSA: Sub-} Saharan Africa; WHO: World Health Organisation

\section{Acknowledgements}

The Authors gratefully acknowledge the funders of this study, namely (1) Consortium for Advanced Research Training in Africa (CARTA). CARTA is jointly led by the African Population and Health Research Center and the University of the Witwatersrand and funded by the Wellcome Trust (UK) (Grant No: 087547/Z/08/Z),(2) The HIV \& Hepatocellular Carcinoma Research Program in Uganda (H2U), a U-54 Consortium grant from National Cancer Institute of the US's National Institutes of Health.

\section{Availability of data and materials}

Datasets used for analysis and writing of this report are available from the corresponding author, on reasonable request.

\section{Author's contributions}

JNM conceptualized the study, participated in collecting data, analysis and writing. PO, FM, LA \& GDK supported conceptualization of the study. PO JA FM \& GDK contributed to analysis and writing of the manuscript. All authors read and approved the final manuscript.

\section{Ethics approval and consent to participate}

This study received approval from Makerere University School of Public Health Higher Degrees, Research and Ethics Committee (IRB Number 00005876; FWA Number 00011353). All eligible study participants went through a consenting process and provided written informed consent in English or one of the local languages (Luganda in central region, and Lugbara in northern region) to participate in the study.

Competing interests

The authors declare that they have no competing interests 


\section{Publisher's Note}

Springer Nature remains neutral with regard to jurisdictional claims in published maps and institutional affiliations.

\begin{abstract}
Author details
'Department of Epidemiology \& Biostatistics, School of Public Health, Makerere University College of Health Sciences, P.O. Box 7072, Kampala Uganda. ${ }^{2}$ Department of Epidemiology, Johns Hopkins Bloomberg School of Public Health, Baltimore, Maryland, USA. ${ }^{3}$ Department of Community Health \& Behavioral Sciences, School of Public Health, Makerere University College of Health Sciences, Kampala, Uganda. ${ }^{4}$ Department of Medicine, School of Medicine, Makerere University College of Health Sciences, Kampala, Uganda. ${ }^{5}$ Department of Medicine, School of Medicine, Johns Hopkins University, Baltimore, Maryland, USA.
\end{abstract}

Received: 30 August 2018 Accepted: 5 December 2018

Published online: 19 December 2018

\section{References}

1. WHO. Hepatitis B. 2018 [cited 2018 20th-07-2018]; Available from: http:// www.who.int/mediacentre/factsheets/fs204/en/.

2. Ott JJ, et al. Global epidemiology of hepatitis B virus infection: new estimates of age-specific HBsAg sero-prevalence and endemicity. Vaccine. 2012;30(12):2212-9.

3. Ringelhan M, McKeating JA, Protzer U. Viral hepatitis and liver cancer. Philos Trans R Soc Lond B Biol Sci. 2017;372(1732):1-11.

4. Yang JD, et al. Hepatocellular Carcinoma Occurs at an Earlier Age in Africans, Particularly in Association With Chronic Hepatitis B. Am J Gastroenterol. 2015:110(11):1629-31.

5. Zampino R, et al. Hepatitis B virus burden in developing countries. World J Gastroenterol. 2015:21(42):11941-53.

6. Bwogi J, et al. Hepatitis B infection is highly endemic in Uganda: findings from a national serosurvey. Afr Health Sci. 2009:9(2):98-108.

7. Ochola E, et al. High burden of hepatitis B infection in Northern Uganda: results of a population-based survey. BMC Public Health. 2013:13(1):727.

8. The Polaris Observatory Collaborators. Global prevalence, treatment, and prevention of hepatitis B virus infection in 2016: a modelling study. Lancet Gastroenterol Hepatol. 2018;3:383-403.

9. Pido B, Kagimu M. Prevalence of hepatitis B virus (HBV) infection among Makerere University medical students. Afr Health Sci. 2005;5(2):93-8.

10. Ziraba AK, et al. Sero-prevalence and risk factors for hepatitis B virus infection among health care workers in a tertiary hospital in Uganda. BMC Infect Dis. 2010;10(191):1471-2334.

11. Bayo $P$, et al. High prevalence of hepatitis $B$ virus infection among pregnant women attending antenatal care: a cross-sectional study in two hospitals in northern Uganda. BMJ Open. 2014:4(11):2014-005889.

12. Pirillo MF, et al. Seroprevalence of hepatitis $B$ and $C$ viruses among HIVinfected pregnant women in Uganda and Rwanda. J Med Virol. 2007;79(12): 1797-801.

13. Kiire CF. The epidemiology and prophylaxis of hepatitis B in sub-Saharan Africa: a view from tropical and subtropical Africa. Gut. 1996;38(2):S5-12.

14. Howell J, Lemoine M, Thursz M. Prevention of materno-foetal transmission of hepatitis B in sub-Saharan Africa: the evidence, current practice and future challenges. J Viral Hepat. 2014:21(6):381-96.

15. Tamandjou CR, et al. Is hepatitis B birth dose vaccine needed in Africa? Pan Afr Med J. 2017:(Suppl 3):1-3.

16. Bodo B, Malande OO. Delayed introduction of the birth dose of Hepatitis B vaccine in EPI programs in East Africa: a missed opportunity for combating vertical transmission of Hepatitis B. The Pan African Medical Journal. 2017; 27(3):19.

17. WHO. Global health sector strategy on viral hepatitis, 2016-2021: towards ending viral hepatitis. 2016 [cited 2018 8th-June-2018]; Available from: http://apps.who.int/iris/bitstream/10665/246177/1/WHO-HIV2016.06-eng. pdf?ua=1.

18. Butt G, McGuinness L, Buller-Taylor T, Mitchell S. Reasons for Nonattendance across the Hepatitis C Disease Course. ISRN Nurs. 2013;2013:579529. Published 2013 Sep 11. https://doi.org/10.1155/2013/579529.

19. WHO, Who Guidelines for the Prevention. Care and Treatment of Persons with Chronic Hepatitis B Infection. Geneva, Switzerland: World Health Organization; 2015
20. Buller-Taylor T, et al. Reducing patient and provider knowledge gaps: An evaluation of a community informed hepatitis C online course. Patient Educ Couns. 2018;101(6):1095-102.

21. USAID. Strengthening Uganda's Systems for Treating AIDS Nationally (SUSTAIN) Report. 2017 [cited 2018 20th-June-2018]; Available from: http:// sustainuganda.org/sites/sustainuganda.org/files/Arua.

22. Uganda Ministry of Health. Annual Health sector performance report 2016-17. 2018 10th-May-2018]; Available from: www.health.go.ug/download/file/fid/1647.

23. Kish L. Survey Sampling, Volume 60 of Wiley Classics Library, A Wiley Interscience Publication, Illustrated Edition, J Wiley, 1965, pp 1-643. Digitized 14th July 2008

24. Uganda Ministry of Health. Hospital and health center survey, 2014. 2015 5th-February-2018]; Available from: http://www.who.int/healthinfo/systems/ SARA_H_UGA_Results_2014.pdf.

25. Kipp W, et al. User fees, health staff incentives, and service utilization in Kabarole District, Uganda. Bulletin of the World Health Organization, 2001(79): p. 1032-1037.

26. Hajarizadeh B, et al. Hepatitis B knowledge and associated factors among people with chronic hepatitis B. Aust N Z J Public Health. 2015:39(6):563-8.

27. Paul T, Marie TP, Bechem E. Knowledge, attitude and practice of staff of 4 hospitals in Yaoundé on the prevention of vertical transmission of hepatitis B. Pan Afr Med J. 2017:28:174, pp1-9. Published 2017 Oct 25. https://doi.org/ 10.11604/pamj.2017.28.174.10971

28. Frambo AA, et al. Prevalence of $\mathrm{HBsAg}$ and knowledge about hepatitis $B$ in pregnancy in the Buea Health District, Cameroon: a cross-sectional study. BMC Res Notes. 2014;7(394):1756-0500.

29. Greenland S. Model-based estimation of relative risks and other epidemiologic measures in studies of common outcomes and in casecontrol studies. Am J Epidemiol. 2004;160(4):301-5.

30. Cook TD. Advanced statistics: up with odds ratios! A case for odds ratios when outcomes are common. Acad Emerg Med. 2002:9(12):1430-4.

31. Global Burden of Disease Liver Cancer Collaboration, Akinyemiju T, Abera S, et al. The Burden of Primary Liver Cancer and Underlying Etiologies From 1990 to 2015 at the Global, Regional, and National Level: Results From the Global Burden of Disease Study 2015. JAMA Oncol. 2017:3(12):1683-691.

32. WHO. Prevention, Care and Treatment of Viral Hepatitis in the African Region: Framework for Action, 2016 - 2020. 2017 17th October 2018]; Available from:

http://afro.who.int/publications/prevention-care-and-treatment-viralhepatitis-african-region-framework-action-2016.

33. Tamayo A, et al. Correlates of disease-specific knowledge among patients with chronic hepatitis B or hepatitis C infection in India. Hepatol Int. 2016 10(6):988-95.

34. Sheng Q-J, et al. Hepatitis B virus serosurvey and awareness of mother-tochild transmission among pregnant women in Shenyang, China: An observational study. Medicine. 2018:97(22):e10931.

35. Abdulai $\mathrm{M}$, et al. Low level of Hepatitis B knowledge and awareness among pregnant women in the Kintampo North Municipality: implications for effective disease control. Ghana Med J. 2016;50(3):157-62.

36. Cunningham SJ, et al. Seroprevalence of hepatitis B and C in a Merseyside hospital for the mentally handicapped. Epidemiol Infect. 1994;112(1):195200.

37. van der Eijk AA, et al. Paired measurements of quantitative hepatitis $B$ virus DNA in saliva and serum of chronic hepatitis B patients: implications for saliva as infectious agent. J Clin Virol. 2004;29(2):92-4.

38. Kidd-Ljunggren $K$, et al. High levels of hepatitis $B$ virus DNA in body fluids from chronic carriers. J Hosp Infect. 2006;64(4):352-7.

39. Heiberg IL, et al. Hepatitis B virus DNA in saliva from children with chronic hepatitis B infection: implications for saliva as a potential mode of horizontal transmission. Pediatr Infect Dis J. 2010;29(5):465-7.

40. Maritz ER, Kidd M, Cotton MF. Premasticating food for weaning African infants: a possible vehicle for transmission of HIV. Pediatrics. 2011;128(3): 2010-3109.

41. Auer-Hackenberg $L$, et al. Short report: premastication in rural Gabon--a cross-sectional survey. J Trop Pediatr. 2014;60(2):154-6.

42. Gaur $\mathrm{AH}$, et al. Prechewing and prewarming food for HIV-exposed children: a prospective cohort experience from Latin America. AIDS Patient Care STDS. 2013 Mar;27(3):142-5. https://doi.org/10.1089/apc.2012.0459.

43. Dun-Dery F, et al. Assessing the knowledge of expectant mothers on mother-to-child transmission of viral hepatitis B in Upper West region of Ghana. BMC Infectious Diseases. 2017;17(1):416. 